\title{
Anti-Spoof Reliable Biometry of Fingerprints Using En-Face Optical Coherence Tomography
}

\author{
Mohammad-Reza Nasiri-Avanaki ${ }^{1,2}$, Alexander Meadway ${ }^{1}$, Adrian Bradu ${ }^{1}$, \\ Rohollah Mazrae Khoshki ${ }^{3}$, Ali Hojjatoleslami ${ }^{2}$, Adrian Gh. Podoleanu ${ }^{1}$ \\ ${ }^{1}$ Applied Optics Group (AOG), School of Physical Sciences, University of Kent, Canterbury, UK \\ ${ }^{2}$ Research and Development Centre, School of Biosciences, University of Kent, Canterbury, UK \\ ${ }^{3}$ Electronic Engineering Department, RAZI University, Kermanshah, Iran \\ E-mail:mn96@kent.ac.uk \\ Received May 22, 2011; revised June 20, 2011; accepted July 5, 2011
}

\begin{abstract}
Optical coherence tomography (OCT) is a relatively new imaging technology which can produce high-resolution images of three-dimensional structures. OCT has been mainly used for medical applications such as for ophthalmology and dermatology. In this study we demonstrate its capability in providing much more reliable biometry identification of fingerprints than conventional methods. We prove that OCT can serve secure control of genuine fingerprints as it can detect if extra layers are placed above the finger. This can prevent with a high probability, intruders to a secure area trying to foul standard systems based on imaging the finger surface. En-Face OCT method is employed and recommended for its capability of providing not only the axial succession of layers in depth, but the en-face image that allows the traditional pattern identification. Another reason for using such OCT technology is that it is compatible with dynamic focus and therefore can provide enhanced transversal resolution and sensitivity. Two En-Face OCT systems are used to evaluate the need for high resolution and conclusions are drawn in terms of the most potential commercial route to exploitation.
\end{abstract}

Keywords: Optical Coherence Tomography, En-Face OCT, Fingerprints, Biometry, High Resolution

\section{Introduction}

Optical coherence tomography (OCT) is an advanced high resolution, non-invasive imaging tool to image the internal structure of skin. OCT can deliver three-dimensional (3D) images from the microstructure compartments within the skin tissue [1,2]. OCT images are produced by measuring the backscattered light from different depths within the tissue.

A commonly employed modality is time domain OCT (TD-OCT) where depth is scanned by adjusting the optical path length in the reference arm of an interferometer using a movable mirror, as illustrated in Figure 1(a). When the optical path lengths of the light returned from the sample and the light in the reference arm match, modulation of the detected signal takes place. The signal produced from a reflector is a series of fringes contained within a Gaussian envelope, the width of which defines the depth resolution of the system. Cross section images can be obtained, as shown in Figure 1(b) and hence 3D volumes can be generated from them.

The amplitude and phase of the back scattered signal obtained from the photodetector are determined based on the interaction of skin compartments with light. From the interference theory, the photodetected signal is:

$$
i=k\left[\rho_{s}+\rho_{r}+2 \sqrt{\rho_{s} \rho_{r}} \Pi \cos \left(\frac{2 \pi}{\lambda_{0}} x\right)\right] \frac{P_{0}}{2}
$$

where $k$ is the photodetector responsivity, $\rho_{s}$ the sample reflectivity, $\rho_{r}$ the reference mirror reflectivity, $\lambda_{0}$ the central wavelength of the optical source and $P_{0}$ the power incident on the object. In terms of the product of depth resolution and penetration depth, OCT fills the gap between confocal microscopy and ultrasound imaging. This product is approximately $0.1 \mu \mathrm{m} \times 500 \mu \mathrm{m}$ in confocal microscopy, $1 \mu \mathrm{m} \times 3000 \mu \mathrm{m}$ in OCT and 50 $\mu \mathrm{m} \times 5000 \mu \mathrm{m}$ in high frequency ultrasound [1].

Traditional biometrics technologies used for fingerprints are based on imaging external features of fingertip 


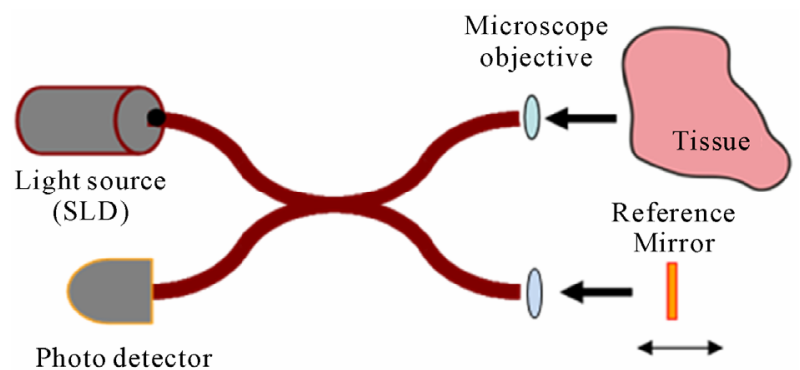

(a)

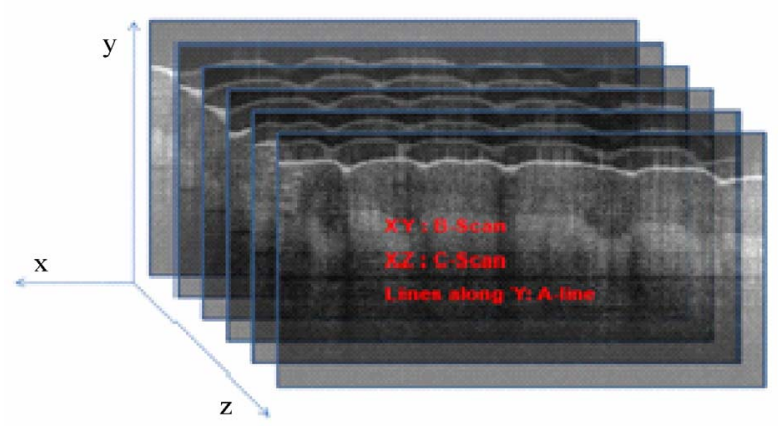

(b)

Figure 1. (a) Simplest setup of OCT based on a Michelson interferometer, (b) OCT cross section images (B-scans) from skin fingertip.

skin [3]. This allows criminals to easily fool and tamper with devices based on traditional methods, by distorting, modifying or counterfeiting the superficial features. For instance, fingerprints can be copied on thin layers attached to the finger or artificial dummies are used. The images produced with conventional cameras represent a 2D map of the object surface and therefore cannot make any difference between a genuine finger and a dummy. Conventional means do not provide any information from inside the finger. This is the aspect which OCT addresses uniquely. The extra depth information provided by OCT has not been translated yet to comercially available fingerprint imaging systems.

OCT has recently been proven as a versatile tool in biometrics [4-6] that can provide enhanced reliability in comparison to conventional imaging methods. Conventional biometrics relies on digital cameras to identify superficial features [7]. With OCT the internal and external features can be extracted from skin, and these can be used to prevent cheating by those intending to use faked fingerprints. In this paper we demonstrate that OCT is capable of producing detailed high resolution cross sections of unusual layer structures of faked fingerprints. In this way, OCT can disclose if any additional material has been added to the skin. Then en-face OCT images can be produced to read the patterns like any digital camera in conventional border control imagers. Additional infor- mation can also be provided such as liveness of the finger or the stress of the subject. The latter two possibilities are not demonstrated here, but they could be implemented based on the recent progress of OCT technology, and these will be discussed in the Conclusions.

\section{Imaging Technology Developed in the Applied Optics Group}

Recently, spectral domain OCT [8] has been proven as a fast imaging technique. However, spectral OCT has disadvantages in terms of high transversal resolution over extended axial depths. Therefore, time domain OCT is better suited for the current application. Different from the conventional time domain OCT, a special en-face $(e F)$-OCT imaging technology is used, developed in the Applied Optics Group. This allows real time images to be produced in sequential regimes, en-face (C-scans) and cross sections (B-scans). Two time domain en-face OCT systems were used to quantify the parameters of an OCT reader machine to image fingerprints, as explained below. These two systems have been further improved for the task of imaging fingerprints, by using different methods to enhance the resolution.

The $(e F)$ OCT method creates images by employing transversal priority scanning (T-scans) [9]. In the $e F-\mathrm{OCT}$, images are generated from many $\mathrm{T}$-scans. These are transversal profiles of reflectivity generated by scanning the optical beam transversally, along different trajectories (raster, helicoidal, etc). eF-OCT offers certain advantages. First, T-scans can be used to generate cross section images (B-scans) as well as coronal plane oriented scans (C-scans) [10]. This allows $e F-O C T$ technology to perform quick alternating imaging sessions in orthogonal planes by instantly switched scanning regimes [11]. Second, the technology is compatible with live and simultaneous generation of a conventional fundus image (coronal-plane oriented) [12]. Third, the $e F-$ OCT is ideal for dynamic focus. While in general, TDOCT is compatible with dynamic focus, it is hard to implement dynamic focus in a traditional OCT system, based on A-scans (axial reflectivity profile). This is because the focus adjustment needs to be synchronized with the A-scanning, which is fast and determined by the line scanning rate. In contrast, with $e F-\mathrm{OCT}$, the demand for the focus adjustment is relaxed, as the focus adjustment needs to be performed at the frame rate, which is of much lower frequency rate. The importance of dynamic focus is expected to become more important in the near future as progress in the adaptive optics (AO) assisted OCT accelerates. When the AO loop manages to achieve ideal correction, A-scan based OCT methods would be limited to collecting signals from a much reduced depth 
range. Therefore, $e F$-OCT turns out to be a better choice for combination with $\mathrm{AO}$, than any other OCT methods.

\section{Detailed Description of the eF-OCT Imaging Systems Used}

\section{1. eF-OCT with Dynamic Focus}

The first system presented uses a dynamic focus scheme, recently assembled in the Applied Optics Group. With the dynamic focus scheme, the coherence gate is synchronous with the focus point determined by the interface optics, hence the transversal resolution is conserved throughout the depth and enhanced signal is returned from all depths. Therefore, images with higher resolution are collected than using standard OCT. The optical components of such a system are shown in Figure 2. A dynamic focus OCT system is especially useful in applications requiring large depth scanning as well as high lateral resolution. Such a system produces C-scan and B-scan images with a lateral image size of up to $5 \mathrm{~mm}$, with transversal resolution better than 5 microns. The system operates at $1300 \mathrm{~nm}$ and uses an SLD, bandwidth $55 \mathrm{~nm}$, which determines a depth resolution in tissue better than 14 microns.

\subsection{Aberration Corrected eF-OCT System Using Adaptive Optics}

The second system uses adaptive optics to eliminate or

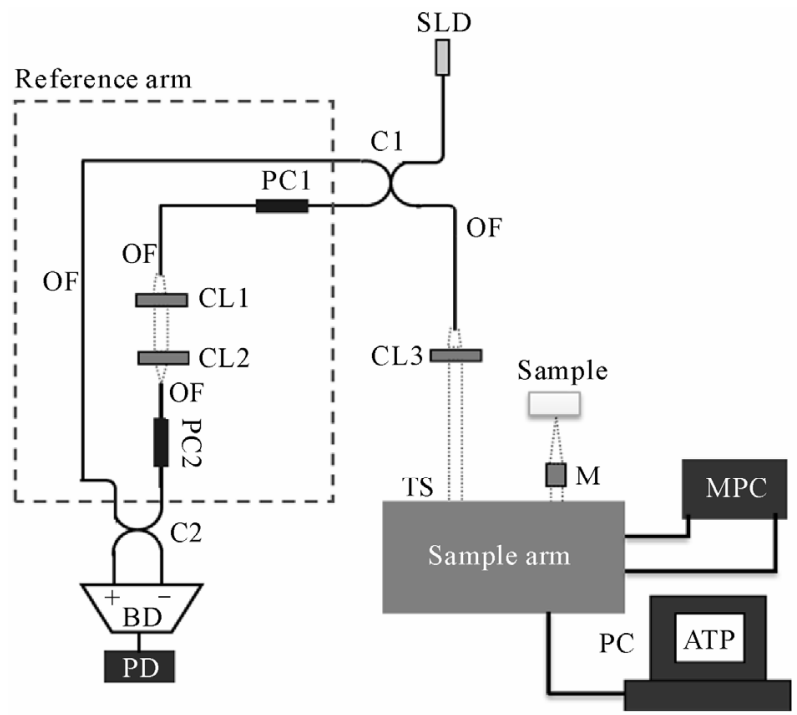

Figure 2. Dynamic focus time domain OCT optical setup. SLD: superluminescent laser diode, PD: photodiode, C1: 2 $\times 2$ coupler, OF: single mode fibre; $P C$ : personal computer, BD: balance detection, CL: Collimator lens, MPC: Mirror positioning controller, MC: Motion controller, PC1, PC2: polarization controllers.

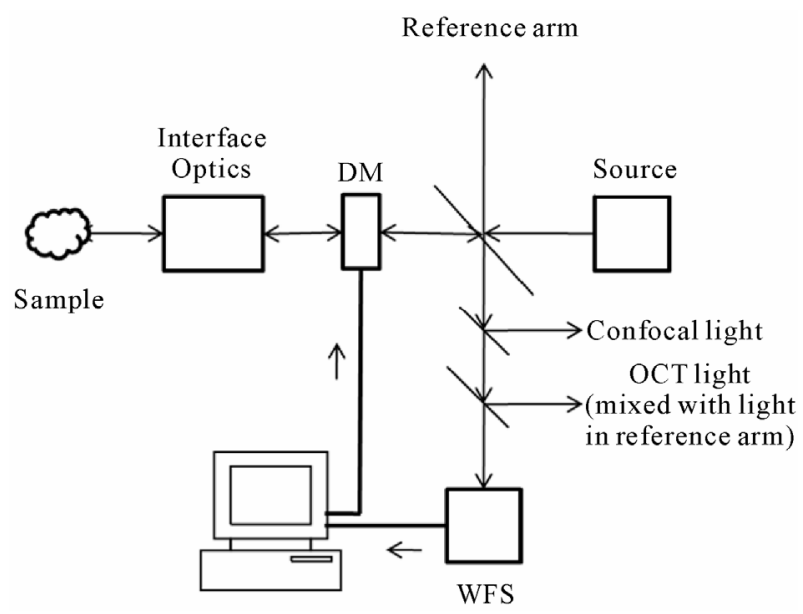

Figure 3. Dual channel OCT/microscope with closed loop adaptive optics.

reduce the aberrations in the system. This system can provide higher resolutions overall. Here, enhanced transversal resolution is targeted. Therefore, the lateral image size is smaller than in the previous system, up to $1 \mathrm{~mm}$. As shown in Figure 3, light from the source (824 nm central wavelength, $20 \mathrm{~nm}$ bandwidth) is split by a beam splitter into the reference and sample arms. Light in the sample arm is incident on the sample and returned towards two imaging channels. The first channel produces a confocal image of the sample, used as a guide for the C-scan OCT images which are produced by a second channel. A Shack Hartman wavefront sensor (WFS) is used to sense the aberrations. The signal from the WFS is processed on a computer which calculates the required shape of the deformable mirror (DM) to correct for the aberrations, in closed loop, optimising the resolution of the system and increasing the intensity of the returned signal. Measurements have shown that transversal resolution below 3 microns can be obtained while the sample is positioned at an axial distance larger than $1 \mathrm{~cm}$ (at such large gaps between the sample object and the microscope objective, much worse resolutions are achievable in micrcopy).

\section{Results and Discussion}

We imaged the fingertip of a volunteer with and without a piece of sellotape on it. Intruders may use thin layers to translate the genuine pattern onto their finger. Such supporting layers can be made from sellotape, wax, gel or any other material.

Our OCT system can produce two types of images, and both are useful for the purpose investigated here: B-scan or cross section images and C-scan or en-face (microscopy orientation). In Figure 4(a) and (b), C-scan OCT images of a fingertip are collected with and without 


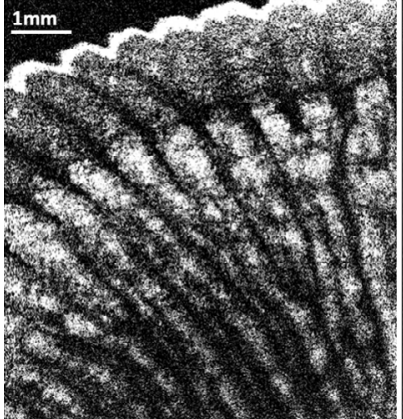

(a)

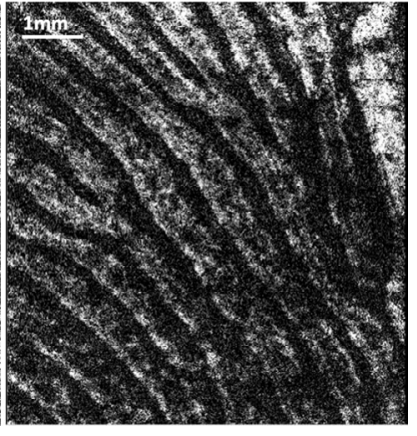

(b)
Figure 4. C-scan images collected with the dynamic focus TD-OCT. (a) without sellotape (b) with sellotape.

sellotape on it, respectively. These images are sampled from different depths, according to the axial position of the coherence gate and this is why they may look differently, however they do not disclose the existence of the sellotape. Images with similar aspect are however collected using conventional systems at the border control points. What we show here is that OCT can explore the fingerprint in depth and this may be an extra feature not utilised so far in security. The pattern is clear and can serve the purpose of identification.

As mentioned above in Section 3, our eF-OCT systems are characterised by the fact that they could easily be switched between $\mathrm{C}$-scan and B-scan regime, by reconfiguring the signals sent to the three scanners: X-lateral,

Y-lateral and Z-depth. The sellotape layer could not be seen in the C-scan images above, however could be traced in cross section images (B-scans). Such B-scan images are shown in Figure 5. Now, the sellotape layer is clearly distinguishable, covering the finger and following its shape.

To obtain more detailed information, the second system was used. C-scan and B-scan OCT images are shown in Figure 6 and in Figure 7 respectively. The sweat ducts are much larger and tiny feature can be better identified. The sellotape layer is clearly seen in the cross section image in Figure 7 (bottom).

Figure 8 shows a graph of two depth profiles obtained from the images in Figure 7. An extra peak in the A-scan profile is identified, marking the axial position of the sellotape. The figure also shows how the addition of a mask (the sellotape) changes the optical properties of the compound sample, made from the sellotape and the finger. The reflection at the surface is reduced, allowing more light to penetrate to the lower layers. Further research may allow interpreting such changes and quantifying the values of the indices of refraction involved.
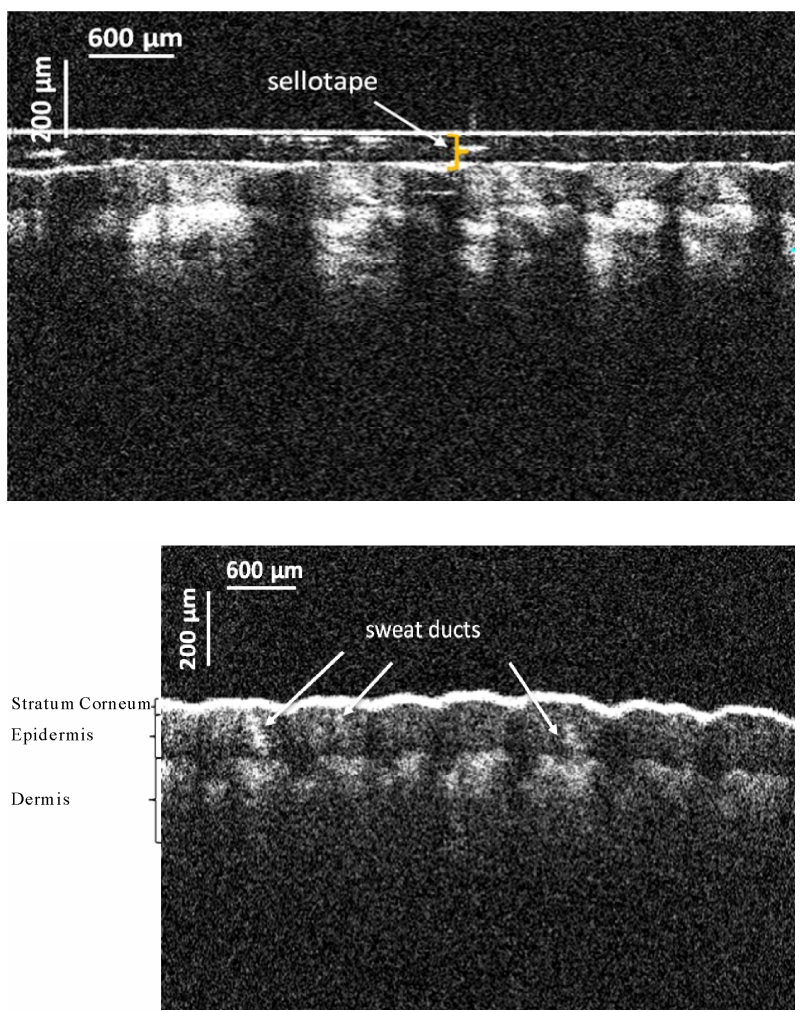

Figure 5. B-scan OCT images collected with the dynamic focus eF-OCT system. (top): without sellotape; (bottom) with sellotape.

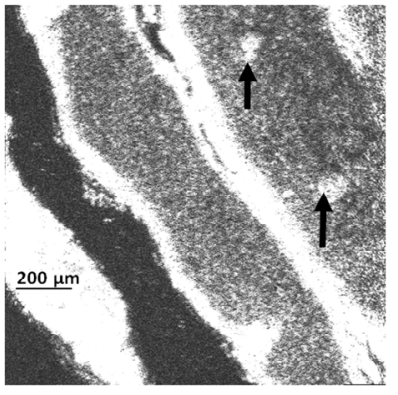

(a)

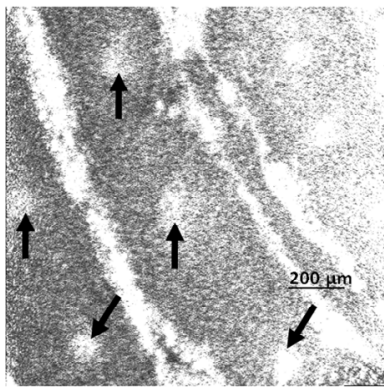

(b)
Figure 6. C-scan images collected with the AO-eF-OCT system. (a) without sellotape; (b) with sellotape. The white blobs shown by the arrows are sweat ducts.

\section{Conclusions}

The two systems above represent state of the art $e F-O C T$. We explored the capability of dynamic focus and adaptive optics to improve the potential of deciphering fake layers among genuine skin layers. The depth of the covering material is disclosed and the thickness of the layer, measured. We have proven above that the two regimes of operation, characteristic to the $e F-\mathrm{OCT}$ only, can advantageously be used to detect any extra layers (using the B-scan regime) and perform the standard operation of 

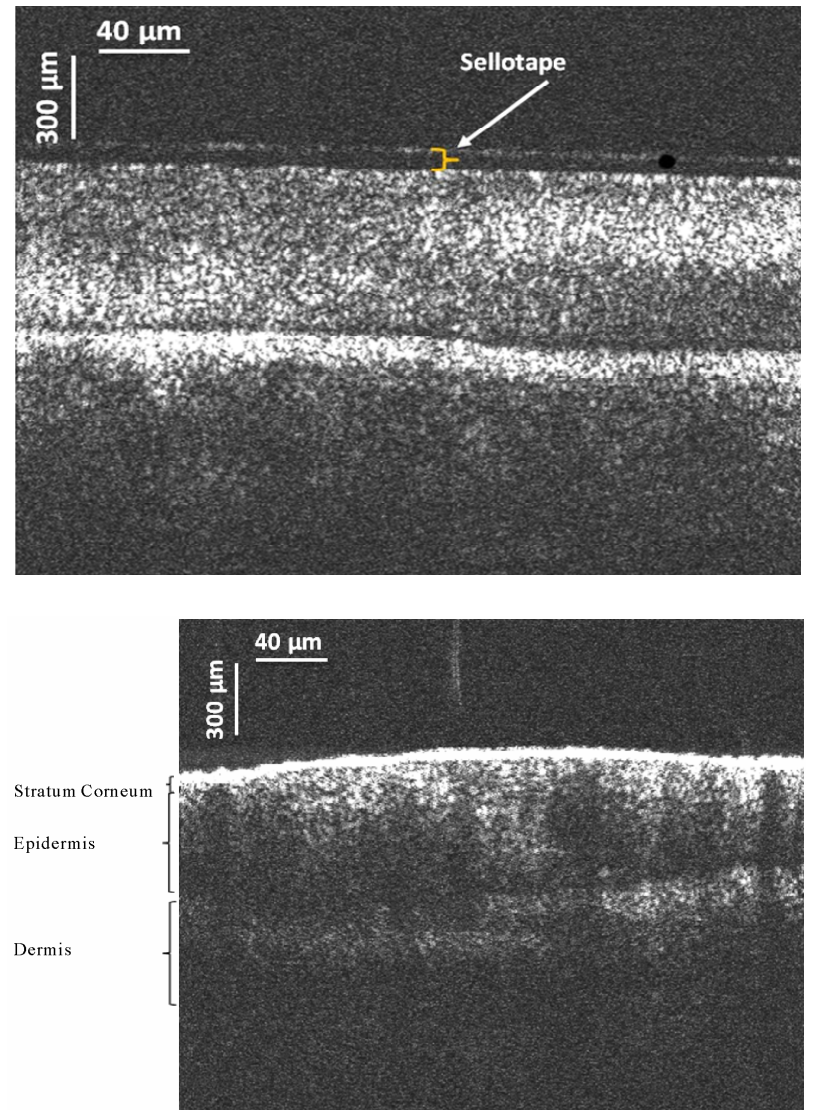

Figure 7. B-scan OCT images collected with the corrected aberration OCT. (top) without sellotape: (bottom) with sellotape.

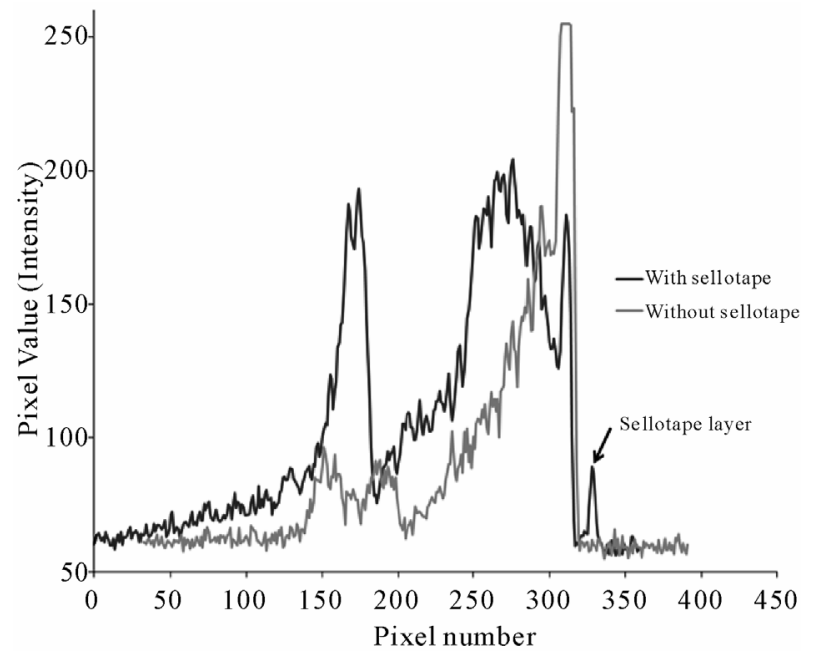

Figure 8. Depth profiles of a thumb, with and without a sellotape mask, obtained from images in Figure 7.

fingerprint recognition (C-scan regime). Their enhanced capabilities in terms of resolution can be extended to other aspects of biometry in order to improve the reliability of security decisions.

\section{Impact on the Technology of Fingerprint Readers}

Conventional means do not provide any information from inside the finger. This is the aspect which OCT addresses uniquely. The extra depth information provided by OCT has not been translated yet to commercially available fingerprint imaging systems.

As opposed to most of the existing biometric methods, based on imaging superficial features, OCT can enhance the reliability of fingerprint imaging. Imaging systems based on OCT eliminate the security flaws and could not be easily spoofed by fingerprint dummies. OCT can extract features in multilayer objects and therefore could be extended to other security applications. OCT can even "see" the genuine fingerprint behind the faked one.

Another possible direction is the liveness detection. The system can be easily modified to perform Doppler OCT, and become sensitive to blood flow. Moving flow can be used as a contrast mechanism. With such a system, the security centre can check the liveness of the finger, and in this way, can prevent cheating by using a dead finger [13]. A dead finger would have no motion within it.

A second possible direction is that in detecting stress. As shown in images above, the sweat ducts are well visible, as white blobs in the C-scans and as helicoidal structures in the B-scans. Usually, an intruder should manifest excess sweetening which could be picked up by OCT when restricting the imaging process to the sweat ducts. There are two types of human sweating: thermal and mental sweating. This investigation process can be applied to both types of sweating. Thermal sweating is stimulated by external heat, while mental sweating occurs in response to mental or physical stress [14]. In response to the stress stimulus, only a small amount of sweat is secreted due to mental sweating. However, it has been proven that OCT can successfully detect such small values of sweat [15] and therefore OCT can complement a "Lie detector testing device"...

To implement OCT technology requires a special source, two or three scanners and an interferometer. In the last 5 years, the cost of OCT systems decreased considerably due to the increase in the numbers of ophthalmology practices employing OCT, as well as due to the expansion of OCT outside ophthalmology. Preventing criminals from accessing restricted places is priceless. This can justify for the moment, investment into adding OCT readers to the conventional recognition machines. The second system, using adaptive optics is still relatively expensive. This was used here to investigate the value of a higher resolution image only and not as a suggestion to expand it towards commercialization. The 
first system however, implementing dynamic focus, is not more expensive than any other OCT system on the market.

We have also shown here, that $e F-\mathrm{OCT}$ can also perform the task of recognition, as it can provide a real time en-face image, similar in its orientation to a microscopy image. Such a system can accomplish booth goals, detect extra layers in top of the finger (in the B-scan regime) and be used in the en-face image collection (in the C-scan regime).

\section{Acknowledgements}

A. Meadway, M. R. Nasiri-Avanaki and A. Bradu acknowledge respectively support of the Ariba Foundation, New York, University of Kent and EPSRC EP/H004963/1.

\section{References}

[1] A. Gh. Podoleanu, "Optical Coherence Tomography," British Institute of Radiology, Vol. 78, No. 935, 2005, pp. 976-988.

[2] A. M. Schmitt, "Principles and Application of Optical Coherent Tomography in Dermatology," Dermatology, Vol. 217, No. 1, 2008, pp. 12-13.

[3] S. Chang, Y. Cheng, K. Larin, Y. Mao, S. Sherif and C. Flueraru, "Optical Coherence Tomography Used for Security and Fingerprint-Sensing Applications," IET Image Processing, Vol. 2, No. 1, 2008, pp. 48-58.

[4] S. K. Dubey, T. Anna, C. Shakher and D. S. Mehta, "Fingerprint Detection Using Full-Field Swept-Source Optical Coherence Tomography," Applied Physics Letters, Vol. 91, No. 18, 2007, Article ID: 181106. doi:10.1063/1.2800823

[5] S. K. Dubey, D. S. Mehta, A. Anand and C. Shakher, "Simultaneous Topography and Tomography of Latent Fingerprints Using Full-Field Swept-Source Optical Coherence Tomography," Journal of Optics A: Pure and Applied Optics, Vol. 10, No. 1, 2008, Article ID: 015307. doi:10.1088/1464-4258/10/01/015307

[6] R. K. Manapuram, M. Ghosn and K. V. Larin, "Identification of Artificial Fingerprints Using Optical Coherence Tomography Technique," Asian Journal of Physics, Vol. 15, 2006, pp. 15-27.
[7] P. Margot and C. Lennard, "Fingerprint detection techniques," Universite de Lausanne, Institut de Police Scientifique et de Criminologie and Switzerland, Lausanne, 1994, p. 190. ISBN 2-940098-01-8

[8] R. Leitgeb, C. K. Hitzenberger, A. Schaefer, J. Reynolds, D. Marks and A. F. Fercher, "Performance of Fourier domain vs. S. Boppart, Real-Time Domaindigital Signal Processing-Based Optical Coherence Tomography," Optics Express, Vol. 11, No. 8, 2003, pp. 889-894. doi:10.1364/OE.11.000889

[9] A. Gh. Podoleanu, G. M. Dobre and D. A. Jackson, "En-Face Coherence Imaging Using Galvanometer Scanner Modulation," Optics Letters, Vol. 23, No. 3, 1998, pp. 147-149.

[10] A. Gh. Podoleanu, M. Seeger, G. M. Dobre, D. J. Webb, D. A. Jackson and F. Fitzke, "Transversal and Longitudinal Images from the Retina of the Living Eye Using Low Coherence Reflectometry," Journal of Biomedical Optics, Vol. 3, No. 1, 1998, pp. 12-20.

[11] C. C. Rosa, J. Rogers, J. Pedro, R. Rosen and A. Podoleanu, "Multi-Scan Time Domain OCT for Retina Imaging," Applied Optics, Vol. 46, No. 10, 2007, pp. 1795 1807. doi:10.1364/AO.46.001795

[12] A. Gh. Podoleanu and D. A. Jackson, "Combined Optical Coherence Tomograph and Scanning Laser Ophthalmoscope," Electronics Letters, Vol. 34, No. 11, 1998, pp. 1088-1090.

[13] A. Schaefer, J. Reynolds, D. Marks and S. Boppart, "Real-Time Digital Signal Processing-Based Optical Coherence Tomography and Doppler Optical Coherence Tomography," IEEE Transactions on Biomedical Engineering, Vol. 51, No. 1, 2004, pp. 186-190. doi:10.1109/TBME.2003.820369

[14] M. Ohmi, M. Tanigawa, A. Yamada, Y. Ueda and M. Haruna, "Dynamic Analysis of Internal and External Mental Sweating by Optical Coherence Tomography," Journal of Biomedical Optics, Vol. 14, No. 1, 2009, Article ID: 014026.

[15] S. Makita, T. Fabritius and Y. Yasuno, "Quantitative Retinal-Blood Flow Measurement with Three-Dimensional Vessel Geometry Determination Using UltrahighResolution Doppler Optical Coherence Angiography," Optics Letters, Vol. 33, No. 8, 2008, pp. 836-838. doi:10.1364/OL.33.000836 